\title{
Numerical statistics of power dropouts based on the Lang-Kobayashi model
}

\author{
Josep Mulet $^{1}$ and Claudio R. Mirasso ${ }^{2}$ \\ ${ }^{1}$ Instituto Mediterráneo de Estudios Avanzados, (CSIC-UIB), E-07071 Palma de Mallorca, Spain \\ ${ }^{2}$ Departament de Física, Universitat de les Illes Balears, E-07071 Palma de Mallorca, Spain* \\ (Received 27 August 1998; revised manuscript received 15 October 1998)
}

\begin{abstract}
The statistics of power dropouts in semiconductor lasers subjected to delayed optical feedback have been numerically investigated using the Lang-Kobayashi model. The data from the numerical simulations have then been used to calculate the probability distribution functions, mean values, and return maps of the time that elapses between dropouts. In addition, the transition from the "low frequency fluctuation" to the "coherence collapse" regime has also been investigated. The numerical simulations compare well with both experimental results, obtained from multilongitudinal mode lasers, and analytical results obtained from other theoretical models. Evidence of "excitability" within the Lang-Kobayashi model is also reported.
\end{abstract}

[S1063-651X(99)16305-0]

PACS number(s): 05.45.-a, 42.65.Sf, 42.55.Px

\section{INTRODUCTION}

It is well known that semiconductor lasers are very sensitive to optical feedback. While small amounts of feedback can be useful in obtaining linewidth reduction [1], reinjection of light levels of $\sim 1 \%$ of the emitted light can give rise to a chaotic output power and a linewidth that increases by a factor of $\sim 10^{3}$. This behavior is commonly referred to as "coherence collapse" (CC) [2]. For similar feedback conditions but injection currents close to the solitary laser threshold, a peculiar behavior, not yet fully understood, can be observed: the low frequency fluctuation (LFF) regime [3]. In the LFF regime the optical power develops repetitive dropouts in which the power falls to almost zero before recovering over microsecond time scales. The recovery process is much longer than any other typical time scale of the system.

The LFF regime has been extensively studied both experimentally and numerically [3-24]. While most of the experiments have been carried out with lasers that lase with several longitudinal modes and undetermined feedback levels, the theoretical and numerical studies have mainly been based on the well known Lang-Kobayashi (LK) model [25]. This model assumes single mode operation of the laser diode subject to weak to moderate external feedback and considers only one reflection within the external cavity.

Recent renewed interest in the LFF and CC regimes has, in part, centered on how the overall dynamics are affected by the multimode nature of many of the lasers investigated. One of the areas in which multimode operation is thought to play a crucial role is in the recovery process of the optical power after a dropout. Recently, Huyet and co-workers [11,12] have experimentally shown (with a Fabry-Perot Hitachi HLP 1400 laser) that after a dropout, and while the output power is still recovering, many of the longitudinal modes of the laser clearly switch on, even though at a later stage, and before the subsequent dropout, the laser operates predominantly in a single longitudinal mode. An important feature of this recovery process is that the different longitudinal modes

*URL: http://formentor.uib.es/Photonics/ of the laser undergo fast pulsations on a 20-100 ps time scale. These fast pulsations have been experimentally observed using streak cameras $[9,13]$. Interestingly, fast pulsations during the recovery process were predicted by the LK model before the experiment's verification [20]. Although there are some discrepancies with respect to the origin of the pulses [9,13], it seems that this behavior is not exclusively related to the multimode character of the laser but is a more general aspect of the dynamics. The LK model predicts that the intensity distribution during the high frequency dynamics has a maximum at very low powers and is monotonically decreasing as the power increases [24]. These numerical results are in good agreement with recent experimental observations [14], but are in contradiction with earlier experimental results, where a distribution function with a maximum at the mean value of the laser power was obtained. Moreover, an asymmetrically decreasing distribution for both low and high power levels was experimentally observed [11]. This behavior was attributed to the multimode character of the laser. However, the results presented in Ref. [11] appear to apply to a special case (high injection current) and cannot be regarded as the generic influence of multimode emission.

Very recently, the coexistence of both the stable operation and LFFs has been observed experimentally in a Hitachi HLP 1400 laser when an etalon was inserted between the laser and the external mirror [17]. This work confirmed an earlier theoretical prediction of the LK model, that one of the external cavity modes of a laser subject to feedback is always stable. This mode is commonly referred to as the maximumgain mode (MGM) [21]. Numerical studies using the LK model have shown that spontaneous jumps from LFF to stable operation, and vice versa, can occur [24].

To gain insight into the LFF regime several studies into the statistics of the time interval $T$ between dropouts have been undertaken. At least four experiments $[6,8,10,16]$ reported the same qualitative results. Namely, that well within the LFF regime the distribution function of $T$ is single peaked, but as the current is increased a multipeaked distribution function develops. The mean time between dropouts $\langle T\rangle$ monotonically decays with increasing current. Although the experiments have been carried out with various diode 
lasers, all of them predominantly exhibit multilongitudinal mode behavior in the LFF and CC regimes.

From the numerical point of view, it is difficult to compute the very long time series required to calculate $\langle T\rangle$. To overcome this problem two different approaches have been taken. On the one hand, the Henry-Kazarinov (HK) theory has been used $[4,8,16]$ to estimate $\langle T\rangle$. This approach assumes that the system lies in a potential well and is forced out of the well by noise at times $T_{0}$. The time $T$ between dropouts is thus $T=T_{0}+T_{r}$, where $T_{r}$ is the recovery time which is usually negligible. In a second approach, Eguía et al. [19] introduced a dynamical model in which the system is excitable and exhibits an Andronov bifurcation. They proposed a simple set of equations and obtained an analytical expression for $\langle T\rangle$. The model developed in [19] is also able to reproduce, at least qualitatively, the experiments of excitability reported in [10].

In this paper extensive numerical simulations of the LK model are performed to evaluate the statistics of the time $T$ between dropouts. To our knowledge, these types of simulations have not been carried out before. The aim of this work is to compare numerical results with previously reported experimental and theoretical results. Moreover, it will be shown that a laser subject to optical feedback and described by the LK model is able to exhibit excitability, under appropriate conditions. The paper is organized as follows: the model is described in Sec. II and the numerical results of the statistics of the time elapses between dropouts are reported in Sec. III. Section IV reports evidence of excitability in the LK model and conclusions are given in Sec. V.

\section{THE MODEL}

A single mode semiconductor laser subject to weak to moderately strong feedback (that is, approximately $10^{-6}$ to $10^{-2}$ of the light emitted is fed back) can be described in terms of the Lang-Kobayashi equations [25],

$$
\begin{aligned}
\frac{d E(t)}{d t}= & \frac{1}{2}(1+\mathrm{i} \alpha)\left[G(N, P)-\Gamma_{0}\right] E(t)+\gamma E(t-\tau) e^{-\mathrm{i} \omega_{0} \tau} \\
& +F_{E}(t) \\
& \frac{d N(t)}{d t}=p \frac{J_{\text {th }}}{q}-\frac{N(t)}{T_{1}}-G(N, P) P(t) \\
& G(N, P)=\frac{\xi\left(N-N_{t}\right)}{1+\epsilon P}, \quad P(t)=|E(t)|^{2}
\end{aligned}
$$

where $E(t)$ is the slowly varying amplitude of the electrical field inside the cavity and $N(t)$ is the carrier number. $\gamma$ and $\tau$ are the feedback rate and the delay time in the external cavity, respectively, $\omega_{0}$ is the solitary laser frequency, $\alpha$ is the linewidth enhancement factor, $p$ is the pump current in units of solitary threshold current $J_{\text {th }}$, and $q$ is the electronic charge. The effect of optical saturation of the active medium is included via the nonlinear gain parameter $\epsilon . \xi$ is the differential gain; $T_{1}$ and $1 / \Gamma_{0}$ are the carrier and photon lifetimes, respectively, and $N_{t}$ is the carrier number at transparency. $F_{E}$ is a Langevin force describing a (Gaussian) white noise process that represents random fluctuations caused by spontaneous emission whose rate is $R=4 N \beta$. Assuming that the system is Markoffian, random forces have zero mean and are $\delta$ correlated in time $\left\langle F_{E}(t) F_{E}^{*}\left(t^{\prime}\right)\right\rangle=R \delta\left(t-t^{\prime}\right)$. The equations are normalized such that $P=|E|^{2}$ is the number of photons inside the cavity. In order to better compare with the experimental data, a set of parameters that approximately correspond to those of the Hitachi HLP 1400 laser, as estimated from experimental data given in Ref. [15], has been used. $\quad \xi=2.76 \times 10^{-6} \mathrm{~ns}^{-1}, \quad \alpha=4, \quad \epsilon=3 \times 10^{-7}, \quad \Gamma_{0}$ $=158 \mathrm{~ns}^{-1}, \quad T_{1}=0.6 \mathrm{~ns}, \quad N_{t}=1.51 \times 10^{8}, \quad$ and $\beta=8$ $\times 10^{-7} \mathrm{~ns}^{-1} . \gamma=35 \mathrm{~ns}^{-1}$ and $\tau=3.3 \mathrm{~ns}$. The solitary laser threshold current is $55 \mathrm{~mA}$ and the feedback gives a threshold reduction of $\sim 12.3 \%$.

The steady-state solutions of the LK equations lead to fixed points created in saddle-node bifurcations as the feedback rate is increased. These fixed points are usually denoted as external cavity modes and antimodes [18]. Modes and antimodes lie on an ellipse in the carrier-frequency phase space. The antimodes are (unstable) saddle points. The stability of the cavity modes is dependent upon, among others things, the effective feedback strength $C=\gamma \tau \sqrt{1+\alpha^{2}}$. In the weak feedback regime (small $C$ ) the modes are stable and the laser operates in the minimum linewidth mode, which is the mode nearest in frequency to the solitary laser frequency [1]. As $C$ is increased many more modes become unstable due to Hopf bifurcations [21]. Physically, the modes tend to show undamped relaxation oscillations, and the laser is observed to jump between many of these unstable modes. For bias currents close to the solitary laser threshold the system can perform LFFs. Within this regime the system tries to move towards the MGM, located near the top of the ellipse. The path of the trajectory is such that it always passes too close to a saddle point, and is thus repulsed towards the center of the ellipse, producing a dropout, before it can reach the MGM. Afterwards, the system again starts on its way towards the MGM ("Sisyphus effect'" [20,22]).

As mentioned previously, it is of interest to evaluate the mean time $T$ between these dropouts. Henry and Kazarinov (HK) estimated $\langle T\rangle$ by making some approximations to the LK model. Their expression for $\langle T\rangle$ is [4]:

$$
\langle T\rangle \approx \frac{\pi}{a\left(1+\frac{4 P}{P_{1}}\right)} \exp \left[\frac{1}{3 b}\left(1-p_{\text {th }}^{\text {feed }}\right)^{3}\left(1+\frac{P_{1}}{4 P}\right)^{3}\right],
$$

where $P_{1}$ is the mean output-power for the current corresponding to the solitary laser threshold in the presence of feedback and $P$ is the mean power that is dependent on the injection current. $\left(1-p_{\text {th }}^{\text {feed }}\right)$ is the threshold reduction induced by the optical feedback, and $a, b$ are two laserspecific constants. It should noted that spontaneous emission noise is essential in this model since $b \rightarrow 0$ when $\beta \rightarrow 0$. The parameters that best fit our numerical results are $a$ $=25 \mathrm{ps}^{-1}$ and $b=6.32 \times 10^{-3}$, but only for currents above the solitary laser threshold [as will be shown in Fig. 4(a)].

\section{NUMERICAL RESULTS}

The LFF process was explained by Sano as an example of a crisis of an attractor, that is to say, the path of the system trajectory in the phase space always collides with an anti- 

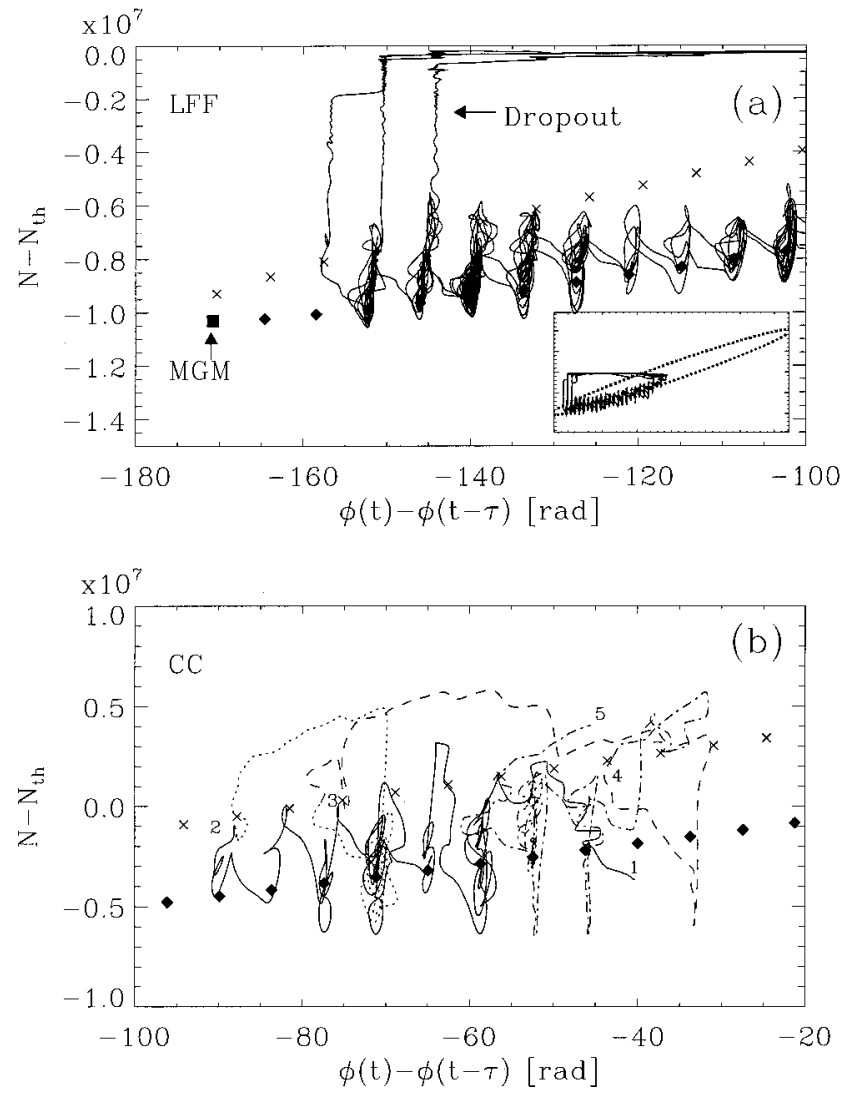

FIG. 1. Evolution of the trajectory in phase space for the (a) LFF and (b) CC regimes. Diamonds denote cavity modes and crosses antimodes; the MGM is denoted with a square. For the LFFs we superposed three dropout events. The system evolves towards the maximum-gain mode passing very close to the antimodes and almost reaching the unstable manifold of the saddle generating a dropout event. In the $\mathrm{CC}$ regime chaos and antimode dynamics compete. The numbers give an idea of the high frequency dynamics involved: 1-2, $10.1 \mathrm{~ns} ; 2-3,3 \mathrm{~ns}$; 3-4, $2.6 \mathrm{~ns}, 4-5,6.2 \mathrm{~ns}$.

mode at some point in its evolution [18]. In Fig. 1(a), we illustrate LFF trajectories in the $\eta-n$ phase space, where $n=N-N_{\text {th }}$ is the carrier number normalized to the solitary laser threshold, and $\eta(t)=\phi(t)-\phi(t-\tau)$ is the phase difference of the slowly varying complex field $E(t)$ in one external round-trip. For comparison, in Fig. 1(b) we illustrate the same kinds of trajectories for the $\mathrm{CC}$ regime. The laser parameters are the same as those described in the previous section, except that $\tau=2.3 \mathrm{~ns}$ and $\gamma=16.5 \mathrm{~ns}^{-1}(5.4 \%$ threshold reduction).

A means of automatically determining whether a dropout event has occurred is a necessity when analyzing long time series. The criterion used in this work makes use of the fact that a dropout event manifests itself in the $\eta-n$ phase space as a sudden large excursion of the system trajectory towards positive values of $\eta$ [see Fig. 1(a)]. A dropout event is assumed to occur when a sudden change of at least six modes in $\eta$, towards the center of the ellipse, is observed. The use of a six-mode scheme to classify a dropout event is somewhat arbitrary, but has been found to consistently distinguish between the chaotic back and forth changes in $\eta$ associated with $\mathrm{CC}$ and a dropout event (at least for the parameter set being considered).

Time series longer than $1 \mathrm{~ms}$ and that averaged over at

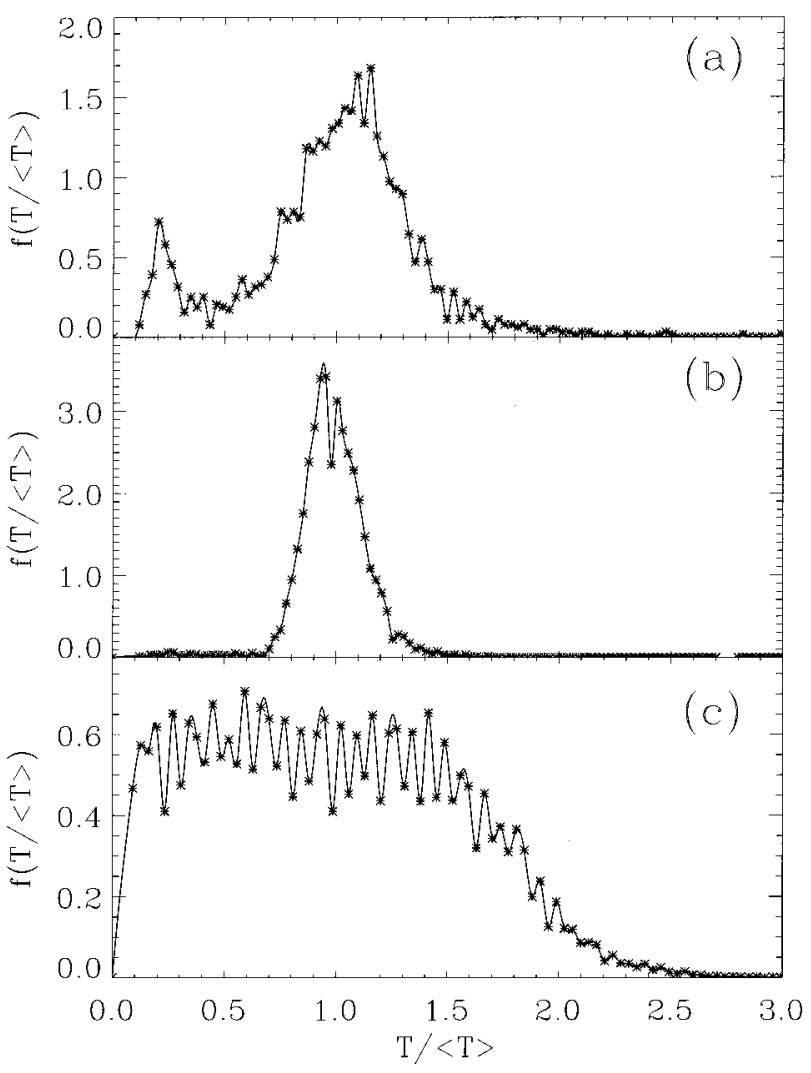

FIG. 2. Probability distribution functions of the time between dropouts for three different bias currents. (a) $p=0.92,\langle T\rangle$ $=312 \mathrm{~ns}$; (b) $p=1.014,\langle T\rangle=116$ ns; (c) $p=1.18,\langle T\rangle=25$ ns.

least $10^{4}$ dropout events were used to calculate the $\langle T\rangle$ for each of the bias currents considered. Although the simulations were performed with spontaneous emission noise, the inclusion of this term seemed to have little effect on the magnitude of the statistical quantities calculated.

Figure 2 shows the probability distribution function (PDF) of the time $T$ between dropouts for three bias currents. These correspond to the LFF regime below the solitary laser threshold, the LFF regime above the solitary laser threshold, and well into the CC regime. As can be seen, in the LFF regime the distribution is nearly single-peaked, in agreement with previous experimental results, obtained from lasers that typically operate with several longitudinal modes $[10,16]$. In Fig. 2(a) a secondary peak appears due to fast excursions between the modes and antimodes when the system is pumped just above the threshold for LFF behavior. Simulations have been undertaken to check that this small peak also appears in the PDF for values of $p$ between 0.88 and 0.92 . For bias currents within the $\mathrm{CC}$ regime the PDF initially has a multipeaked structure that then slowly decays exponentially, and hence is in qualitative agreement with experimental observations as outlined in $[10,16]$. However, the amplitudes of the oscillations in the PDF are very small, when compared to those seen experimentally in Ref. [10]. This seems to indicate that some additional phenomenon needs to be included in the LK model in order to fully describe the features observed experimentally. A phase-locked behavior due to multiple external reflections is the most likely candidate, since it has been noted that reducing the feedback 


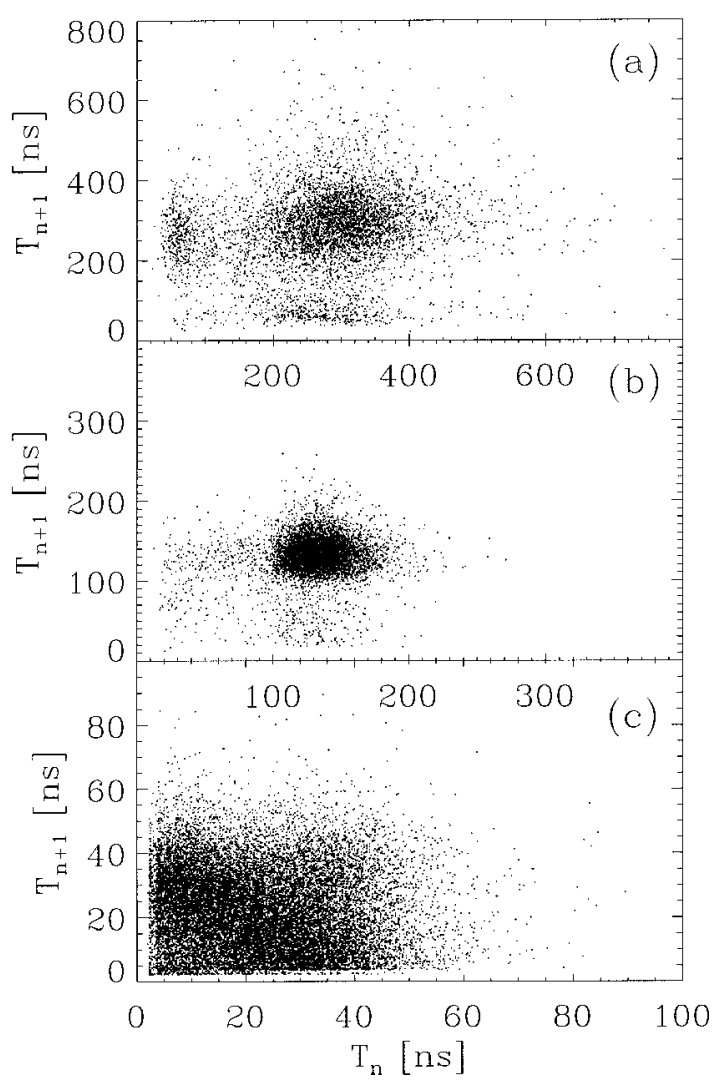

FIG. 3. Return maps $T_{n+1}$ vs $T_{n}$ for the time between dropouts for the same currents as Fig. 2.

strength washes out the oscillations seen in the PDF.

Return maps $T_{n+1}$ vs $T_{n}$ for the currents used in Fig. 2 are shown in Fig. 3. A more or less uniform cloud of points in both the LFF and CC regimes can be seen, indicating a lack of periodicity in the dynamical evolution, with no correlation between dropouts. Qualitatively the same return maps are obtained when the spontaneous emission noise term in Eq. (1) is removed. These results are in good agreement with previously reported experimental data [10], except that the LK model is unable to reproduce the observed substructure that appears in the CC regime. However, our interpretation of the return maps is different from that given in Ref. [10]. The latter interprets LFFs as a consequence of the existence of an Andronov bifurcation in which noise is essential to anticipate the bifurcation and avoid a periodic regime.

Finally, in Fig. 4 we plot the mean time between dropouts vs bias current normalized to (a) the solitary threshold and (b) the threshold of the appearance of LFFs, which in this case almost coincides with the threshold of the laser with feedback. We define here $\epsilon_{1}=p-1$ and $\epsilon_{2}=p / p_{\text {th }}^{\mathrm{LFF}}-1$. The last points of Fig. 4(a), close to CC, can be fitted with $1 / \epsilon_{1}$. This dependence was experimentally found by Sacher $e t$ al. [6]. However, this dependence has been observed to twist for lower currents, in good agreement with more recent experimental results $[8,16]$.

The LK model predicts that stable emission will be obtained for control parameter values less than some critical value $p_{\text {th }}^{\mathrm{LFF}}$. When the control parameter slightly exceeds the critical value, stable emission is interrupted by occasional power dropouts. Hence, the natural threshold required to un-
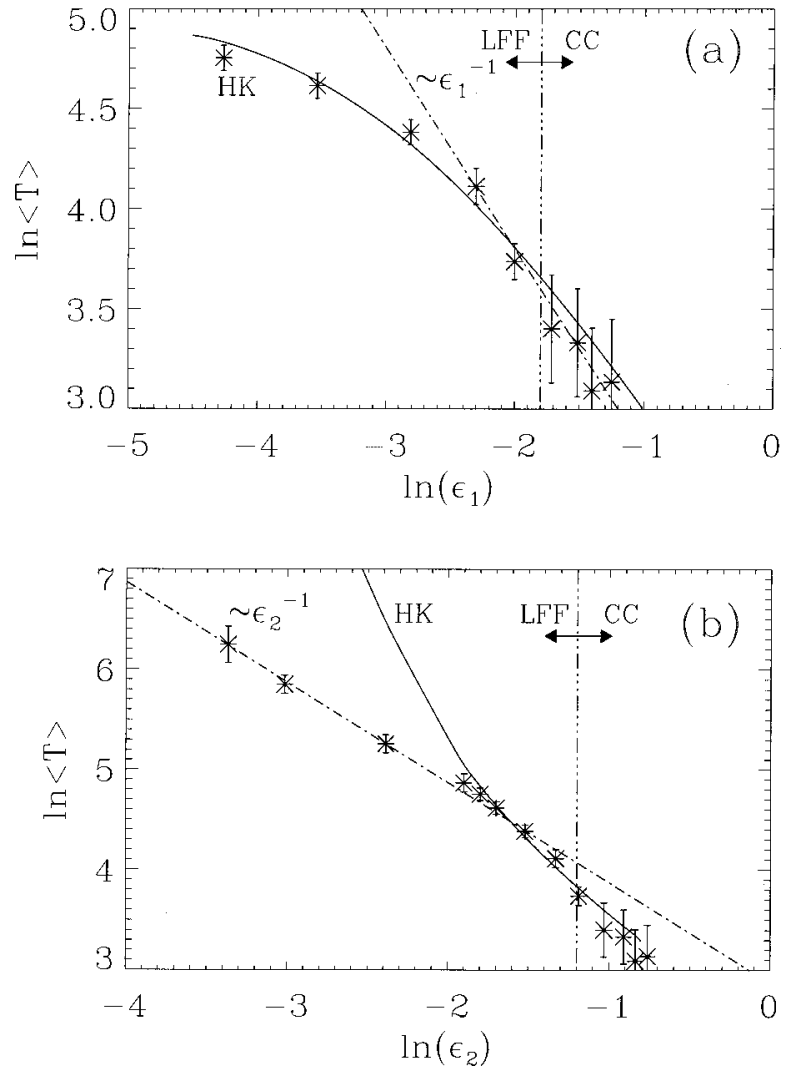

FIG. 4. Mean time between dropouts versus normalized current (a) to solitary laser threshold and (b) to the threshold of the appearance of LFF $\left(p_{\mathrm{th}}^{\mathrm{LFF}}\right)$. Points are calculated from numerical simulations of the LK model. Solid lines correspond to the HenryKazarinov approximation and dot-dashed lines are straight lines with slope -1 . Three dot-dashed lines roughly delimit the LFF and $\mathrm{CC}$ regimes.

derstand the scaling properties of the system appears to be the $p_{\text {th }}^{\mathrm{LFF}}$ and thus the data should be normalized to this value. However, the exact value of $p_{\text {th }}^{\mathrm{LFF}}$ is not easy to find numerically and has been estimated to be $p_{\mathrm{th}}^{\mathrm{LFF}}=0.88 \pm 0.01$. The numerical results in the LFF regime scale very closely to $1 / \epsilon_{2}$ [Fig. 4(b)] when replotted using $\ln \langle T\rangle$ vs $\ln \epsilon_{2}$. Figure 4(b) shows that the HK theory (solid line) cannot be fitted to these data.

The plot of $\ln \langle T\rangle$ vs $\ln \epsilon_{1}$ obtained from the theoretical model proposed by Eguía et al. [19] is only in qualitative agreement with the numerical results presented here and those of Refs. [8] and [16], if the $\epsilon_{\text {threshold }}$ of Eguía's model is interpreted as the threshold of the solitary laser.

\section{EXCITABILITY}

Eguía et al. [19] introduced a dynamical model that predicts that LFFs are induced by noise. Their model has dimension $2(x, y)$ and codimension $2\left(\epsilon_{1}, \epsilon_{2}\right)$. Three fixed points exist for some regions of the $\epsilon_{1}-\epsilon_{2}$ space: a saddle, a node, and a third point that always exists. The node is always located to one side of the saddle, and their relative distance decreases when $\epsilon_{1}$ is increased until the node and the saddle collide and disappear (Andronov bifurcation). In the region in which the third point is spiraling unstable, and only the 


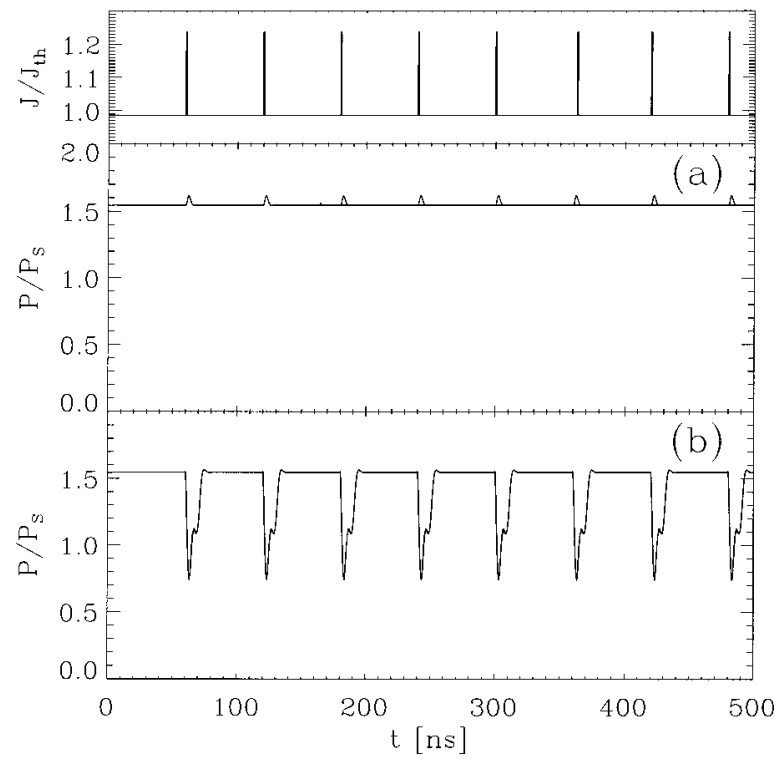

FIG. 5. Time trace of the optical power normalized to the power of the saddle point and filtered with a lower-pass filter of bandwidth $1 \mathrm{GHz}$. The time trace exhibits characteristics indicative of excitability. The amplitudes of the input pulses are (a) $\Delta p=0.05$ (corresponding to $2.75 \mathrm{~mA}$ ) and (b) $\Delta p=0.25$ (corresponding to 13.75 $\mathrm{mA}$ ). The input current is plotted on the top of panel (a).

node is stable, a dropout event is generated when noise drifts the trajectory towards the unstable manifold of the saddle. They define the threshold as the value of the control parameter for which no dropouts occur in one integration time. The excitability phenomenon can be also explained by this model in terms of crossing the Andronov bifurcation.

In our analysis excitability is taken to mean that the system can only develop dropouts if short input pulses are superimposed onto a bias current for which the laser would otherwise be stable. At least three ingredients are required by any system before it can be described as being excitable: first, the existence of a threshold above which an excitation can occur; second, the form and size of the response must be invariant to any change in the magnitude of the perturbation; third, a refractory time must exist (if a second perturbation is applied at a time shorter than the refractory time, the system no longer responds).

Numerically investigations of the LK model have revealed the first evidence, to our knowledge, of excitability in Fig. 5. The parameters used are the same as those described in Sec. II, except that the $\alpha$ factor was lowered to increase stability, $\alpha=3.5$. Values of $\tau=0.6 \mathrm{~ns}, \gamma=10 \mathrm{~ns}^{-1}$ were used and noise was neglected. A train of rectangular pulses of period of $60 \mathrm{~ns}$ was superimposed onto a bias current of $p_{\text {bias }}=0.986$. The rectangular wave had a duty cycle of $0.1 \%$ and amplitude $\Delta p=\left(J_{\text {on }}-J_{\text {bias }}\right) / J_{\text {th }}$, where $J_{\text {on }}$ is the peak of the injection current and $J_{\text {bias }}=p_{\text {bias }} J_{\text {th }}$. Stable emission is obtained for small perturbations [Fig. 5(a)]. When the amplitude of the perturbation is increased the system starts to exhibit dropout events [Fig. 5(b)]. The transition was found to occur when $\Delta p \sim 0.19$. The size and shape of the dropouts were found to be almost invariant to further increases in $\Delta p$.

Numerical simulations have been undertaken to check for the existence of a refractory time in order to verify that the

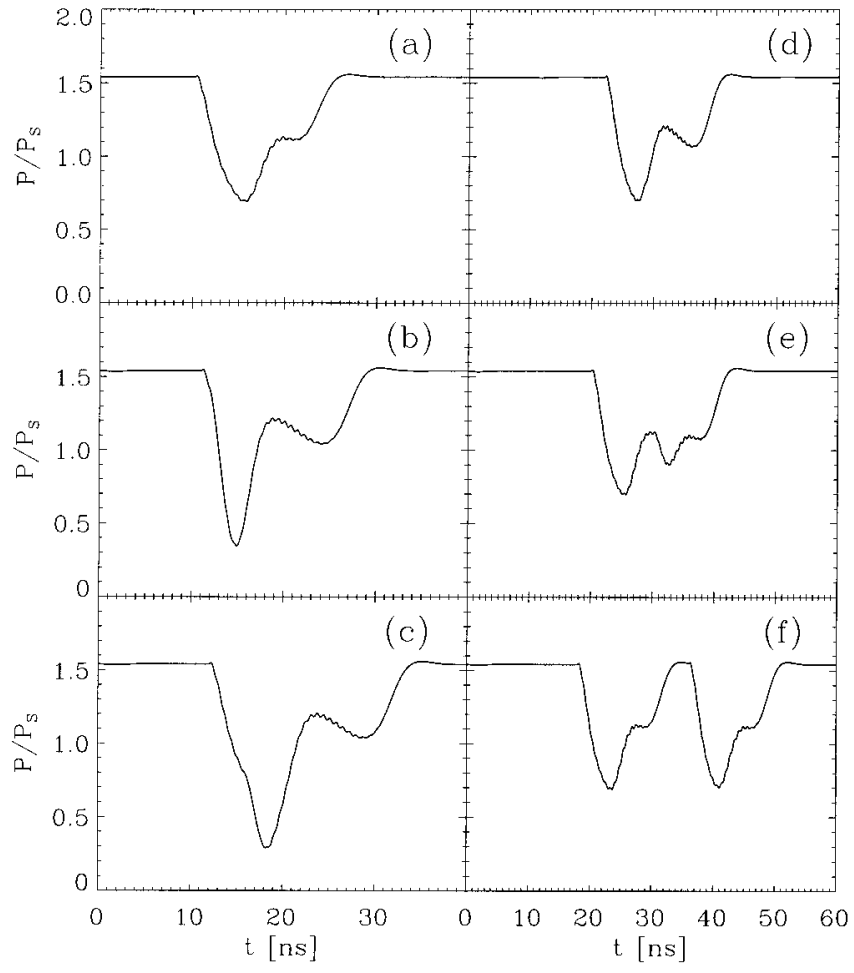

FIG. 6. Response of the system to a couple of pulses with amplitude $\Delta p=0.25$. In (b)-(f) we have increased the time separation $\Delta T$ between the two pulses: (a) a solitary perturbation, (b) $\Delta T$ $=1 \mathrm{~ns},(\mathrm{c}) \Delta T=4 \mathrm{~ns},(\mathrm{~d}) \Delta T=10 \mathrm{~ns}$, (e) $\Delta T=12 \mathrm{~ns}$, and (f) $\Delta T=18$ ns.

model is indeed excitable. The response of the system when a second pulse is injected at a delay time $\Delta T$ with respect to the first pulse is shown in Fig. 6. It is clear that the response does not significantly change for $\Delta T \leqq 8 \mathrm{~ns}$. Increasing $\Delta T$ further to $\Delta T \geq 10 \mathrm{~ns}$ induces significant distortion. The system is unable to respond to the stimulus. The system regains its ability to respond to both pulses when $\Delta T \geqslant 18 \mathrm{~ns}$. It would thus appear that the refractory time is $\sim 10 \mathrm{~ns}$.

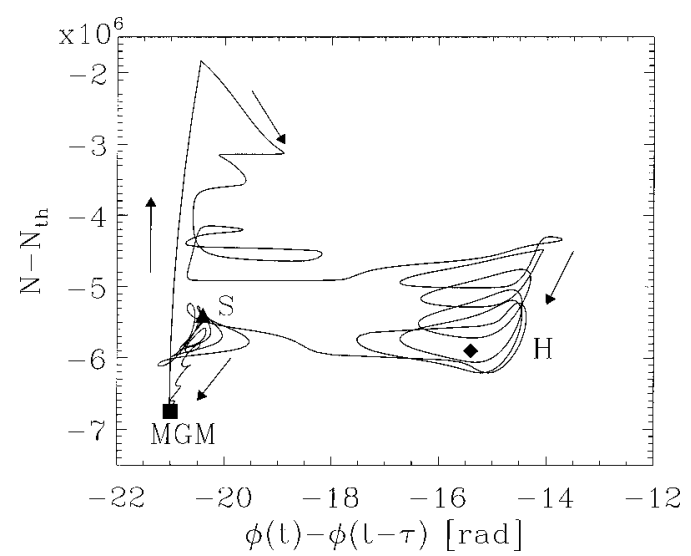

FIG. 7. Phase-space evolution of the trajectory under the excitable condition of $\Delta p=0.25$. The system operates in the MGM. A dropout occurs after which the system goes back to the MGM. The modes and antimodes are calculated for $p=0.986$ for which stable emission is observed. $\mathrm{H}$ is a Hopf-bifurcated external cavity mode, $\mathrm{S}$ is an antimode, and MGM is the maximum gain mode. 
The $\eta-n$ phase space has also been used to investigate the dynamical evolution of the LK excitable scenario. Figure 7 shows the trajectory in this phase space for $\Delta p=0.25$. The pulses excite the system and the trajectory moves towards the saddle point through the unstable manifold, causing the trajectory to move towards the Hopf bifurcated point; a dropout is produced in the system, and at some later time the system returns to the MGM.

\section{CONCLUSIONS}

In conclusion, extensive numerical simulations of a single (longitudinal) mode semiconductor laser subject to optical feedback have been performed. The Lang-Kobayashi model has been used to calculate the statistical properties of the time between dropouts. Bias currents ranging from those associated with low frequency fluctuations to those associated with the coherence collapse regime have been investigated. The probability distribution functions, the mean time between dropouts, and the return maps are all in good agreement with experimental results, which were obtained with lasers that mainly operate with multiple longitudinal modes in these regimes. When the mean time between dropouts vs the injection current (normalized to the threshold of the ap- pearance of the low frequency fluctuations) is plotted using a ln-ln scale, a linear dependence, with slope very close to -1 , was obtained. This is in agreement with experimentally observed behavior for a diode laser subject to optical feedback. The numerical results also suggest that, at least for these types of quantities, the assumption of an intrinsically single mode laser is sufficient to explain the experimental results obtained with lasers that mainly operate with several longitudinal modes.

It has also been shown that the Lang-Kobayashi model is able to reproduce an excitable scenario, under appropriate conditions. Excitability in a semiconductor laser subject to optical feedback has been experimentally reported recently.

\section{ACKNOWLEDGMENTS}

This work has been funded by the Comisión Interministerial de Ciencia y Tecnología, Spain, under Project No. TIC97/0420. J.M. was funded by the Ministerio de Educación y Cultura. Fruitful discussions with J. Tredicce, I. Fischer, T. Heil, S. Balle, and R. Roy are acknowledged. We also acknowledge Paul Spencer for a careful reading of the manuscript.
[1] K. Petermann, Laser Diode Modulation and Noise (Kluwer Academic, Dordrecht, 1988).

[2] D. Lenstra, B. H. Verbeek, and A. J. den Boef, IEEE J. Quantum Electron. QE-21, 674 (1985).

[3] Ch. Risch and C. Voumard, J. Appl. Phys. 48, 2083 (1977).

[4] C. H. Henry and R. F. Kazarinov, IEEE J. Quantum Electron. QE-22, 294 (1986).

[5] J. Mork, B. Tromborg, and P. L. Christiansen, IEEE J. Quantum Electron. QE-24, 123 (1988).

[6] J. Sacher, W. Elsässer, and E. O. Göbel, Phys. Rev. Lett. 63, 2224 (1989).

[7] J. Mork, B. Tromborg, and J. Mark, IEEE J. Quantum Electron. QE-28, 93 (1992).

[8] A. Hohl, H. J. C. van der Linden, and R. Roy, Opt. Lett. 20, 2396 (1995).

[9] I. Fischer, G. H. M. van Tartwijk, A. M. Levine, W. Elsässer, E. Göbel, and D. Lenstra, Phys. Rev. Lett. 76, 220 (1996).

[10] M. Giudici, C. Green, G. Gianconelli, U. Nespolo, and J. R. Tredicce, Phys. Rev. E 55, 6414 (1997).

[11] G. Huyet, S. Hegarty, M. Giudici, B. De Bruyn, and J. G. McInerney, Europhys. Lett. 40, 619 (1997).

[12] G. Huyet, S. Balle, M. Giudici, C. Green, G. Giancomelli, and J. R. Tredicce, Opt. Commun. 149, 341 (1998).

[13] G. Vaschenko, M. Giudici, J. J. Rocca, C. S. Menoni, J. R.
Tredicce, and S. Balle, Phys. Rev. Lett. 81, 5536 (1998).

[14] I. Fischer, T. Heil, M. Münkel, and W. Elsässer, Proc. SPIE 3283, 571 (1998).

[15] S. Hegarty, G. Huyet, P. Porta, and J. G. McInerney, Opt. Lett. 23, 1206 (1998).

[16] D. W. Sukow, J. R. Gardner, and D. J. Gauthier, Phys. Rev. A 56, R3370 (1997).

[17] T. Heil, I. Fischer, and W. Elsässer, Phys. Rev. A 58, R2672 (1998).

[18] T. Sano, Phys. Rev. A 50, 2719 (1994).

[19] M. C. Eguía, G. Mindlin, and M. Giudici, Phys. Rev. E 58, 2636 (1998).

[20] G. H. M. van Tartwijk, A. M. Levine, and D. Lenstra, IEEE J. Sel. Top. Quantum Electron. 1, 466 (1995).

[21] A. M. Levine, G. H. M. van Tartwijk, D. Lenstra, and T. Erneux, Phys. Rev. A 52, R3436 (1995).

[22] C. R. Mirasso, M. Mulder, H. J. W. Spoelder, and D. Lenstra, Comput. Phys. 11, 282 (1997).

[23] P. S. Spencer and A. Shore, Quantum Semiclassic. Opt. 9, 819 (1997).

[24] J. Mulet, Master thesis, Universitat de les Illes Balears, 1998 (unpublished).

[25] R. Lang and K. Kobayashi, IEEE J. Quantum Electron. QE16, 347 (1980). 